\title{
Fijación temprana del tórax inestable: reporte de caso
}

\section{Early fixation of the flail chest: case report}

\author{
Brandon S. Aparicio-Blanco*, Rodolfo Barrios-Del Río, Luis F. Cabrera, Sebastián Sánchez-Ussa, \\ Sebastián Martínez, Adriana Serna-Lozano, Marcia Santafé-Guerrero y Mauricio Pedraza-Ciro \\ Departamento de Cirugía General, Universidad El Bosque, Bogotá, Colombia
}

\section{Resumen}

Antecedentes: En 1926, Jones y Richardson describen por primera vez el tórax inestable. Desde entonces existe gran controversia sobre el manejo conservador comparado con el manejo quirúrgico. Objetivo: Dar a conocer los beneficios, las indicaciones y las contraindicaciones de la fijación temprana de fracturas costales en el tórax inestable. Conclusión: La reducción abierta y la fijación temprana de fracturas costales en pacientes con tórax inestable ha demostrado ser un método útil, efectivo y seguro.

Palabras clave: Fijación interna. Fractura costal. Tórax inestable. Trauma de tórax.

\begin{abstract}
Background: In 1926, Jones and Richardson described flail chest for the first time. Since then, exist great controversy about conservative vs surgical approach. Objective: Define the benefits, indications and contraindications of the early fixation of rib fractures in flail chest. Conclusion: The open reduction and early fixation of rib fractures for patients with flail chest has proven to be a useful, effective and safe method.
\end{abstract}

Key words: Internal fixation. Rib fracture. Flail chest. Chest trauma.

\section{Introducción}

El tórax inestable fue descrito por primera vez en 1926 por Jones y Richardson', quienes lo definieron como el resultado de un trauma contuso, en su gran mayoría en la región anterolateral del tórax, con dos o más fracturas de dos o más arcos costales consecutivos ${ }^{1,2}$.

El $25 \%$ de las muertes de los pacientes politraumatizados son secundarias al compromiso grave del tórax, y de ellos, el 39\% presentan fracturas costales $^{2-4}$. El tórax inestable se presenta en el $5-10 \%$ de los pacientes con trauma cerrado en la región torácica y llega a tener una mortalidad de hasta el $40 \%$ incluyendo las lesiones asociadas ${ }^{4,5}$.

Existe gran controversia sobre el manejo conservador en comparación con el manejo quirúrgico para el tórax inestable 4 . Por ello, nos permitimos presentar un reporte de caso de cirugía temprana del tórax inestable y realizar una revisión crítica de la literatura.

\section{Correspondencia:}

*Brandon S. Aparicio-Blanco 


\section{Caso clínico}

Paciente varón de 54 años que ingresa remitido a urgencias del Hospital Santa Clara en Bogotá, Colombia, por trauma cerrado de tórax de alto impacto secundario a una caída de gran altura. Las radiografías iniciales demostraron fracturas del segundo al sexto arcos costales izquierdos, fracturas diafisiarias de cúbito y radio homolaterales desplazadas, con necesidad de soporte ventilatorio y toracostomía cerrada izquierda (con drenaje de $1300 \mathrm{ml}$ serohemático en las últimas 24 horas). Presenta frecuencia cardiaca de 96 latidos por minuto, presión arterial de 152/74 mmHg y saturación de oxígeno del 93\%. En la inspección del tórax se observa deformidad izquierda con edema generalizado de la pared torácica, disminución de los ruidos respiratorios y movimiento paradójico de la caja torácica. La evolución hemodinámica es estable, pero se hace necesaria la ventilación asistida dada la deficiente mecánica ventilatoria y el mal control del dolor a pesar del tratamiento analgésico multimodal.

Se decide llevar a cirugía para osteosíntesis temprana con planeamiento preoperatorio con tomografía computarizada de tórax y reconstrucción tridimensional (Fig. 1) para caracterización de las fracturas y las estructuras intratorácicas. Se realizó la fijación de las fracturas costales con material de osteosíntesis y colocación de una malla de baja densidad de polipropileno, considerando la pérdida de tejidos blandos que podría condicionar una futura hernia pulmonar posoperatoria (Figs. 2 y 3 ).

La evolución fue satisfactoria, con extubación exitosa a las 24 horas, pronto egreso de la unidad de cuidados intensivos ( $\mathrm{UCl}$ ) hacia una habitación en planta, con buen control del dolor, deformidad corregida y sin complicaciones médicas ni quirúrgicas asociadas.

\section{Discusión}

El tórax inestable se define actualmente como la pérdida de la continuidad de un segmento óseo con la pared torácica, ya sea por diversas fracturas costales o por separación costocondral ${ }^{6}$, que lleva a una respiración superficial y movimientos paradójicos de un segmento de la caja torácica (signo clínico patognomónico) $)^{2,6}$. El resultado es una afectación en la mecánica ventilatoria que pone en peligro la vida del paciente, principalmente cuando se asocia a contusión pulmonar, rotura diafragmática, neumotórax o hemotórax ${ }^{3,7}$.

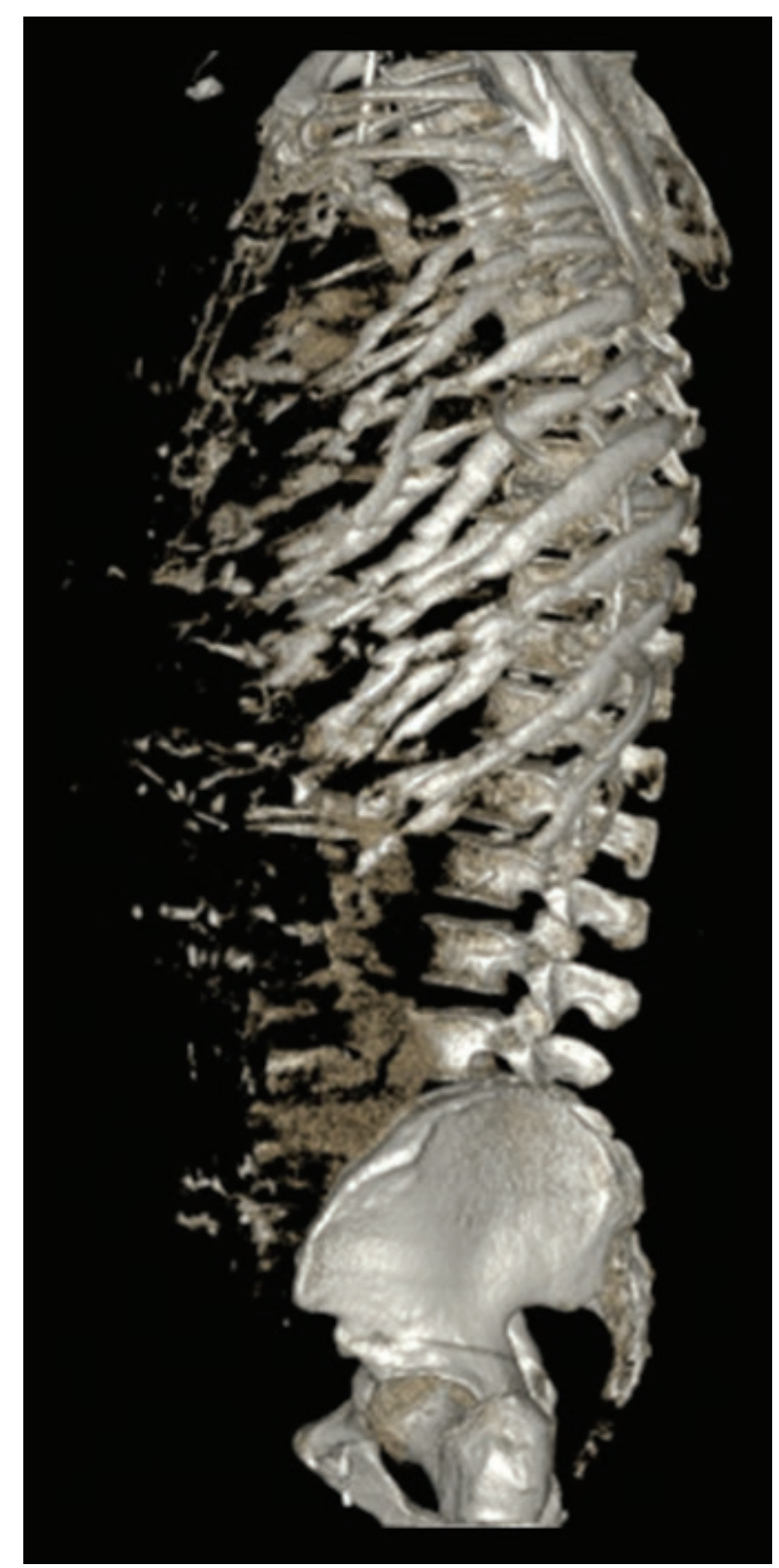

Figura 1. Reconstrucción tridimensional en la que se evidencian fracturas del segundo al sexto arcos costales izquierdos anteriores.

El manejo conservador consiste principalmente en manejar de manera adecuada el dolor con estrategias de analgesia multimodal que incluyen antiinflamatorios no esteroideos (AINE), opiáceos, bloqueos intercostales y colocación de catéteres epidurales; estos últimos tienen los mejores resultados clínicos en cuanto al control del dolor ${ }^{2,7}$. Las medidas de soporte consisten en mantener una buena higiene bronquial, incentivo respiratorio, oxígeno suplementario e intubación orotraqueal y ventilación con presión positiva en casos indicados ${ }^{1,2,7}$. A pesar de estas medidas terapéuticas, se pueden presentar barotrauma, 


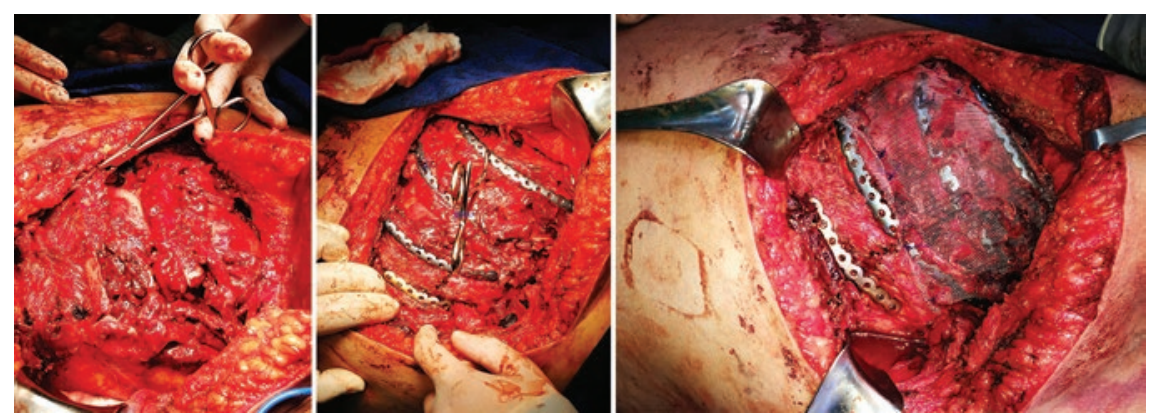

Figura 2. A: disección e identificación de las fracturas costales, exponiendo los arcos costales a fijar. B: fijación abierta de fracturas costales con material de osteosíntesis bicortical. C: colocación de malla sintética no absorbible de baja densidad de polipropileno.

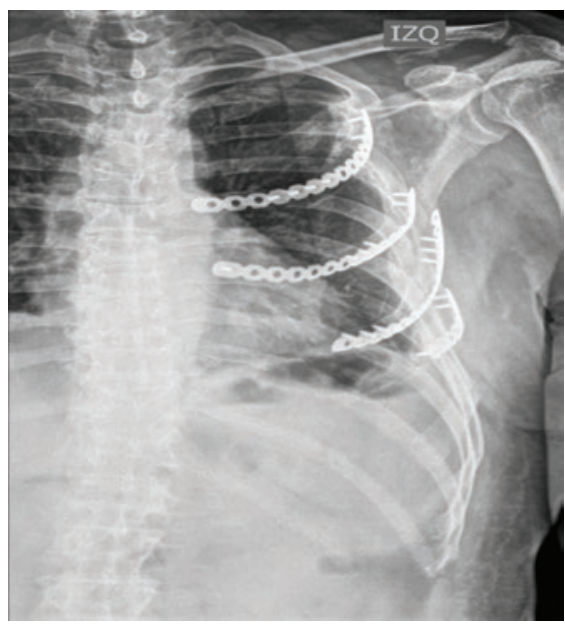

Figura 3. Radiografía en el posoperatorio inmediato.

intubación orotraqueal prolongada, neumonía, infección asociada al ventilador y mayor tiempo de estancia hospitalaria y en la $\mathrm{UCl}^{2,4,8}$. Otras complicaciones asociadas incluyen la deformidad permanente del tórax, el dolor crónico, la dificultad respiratoria y el retraso en el reintegro laboral ${ }^{4}$.

Los pacientes tratados de forma conservadora suelen consultar por dolor persistente asociado a sensación de chasquido, porque las fracturas aún no se han consolidado y este proceso debe suceder en un periodo de alrededor de 90 días. Si esta situación persiste en el tiempo se puede considerar un fallo en la unión, hecho que puede ser ocasionado por múltiples factores, como deficiencia de vitamina $D$, malnutrición, diabetes, uso de corticoides o AINE, y consumo de alcohol o tabaquismo ${ }^{9}$. Por todo lo anterior y con el advenimiento de nuevos materiales de osteosíntesis, se han desarrollado diferentes técnicas de fijación quirúrgica temprana de las fracturas costales en el tórax inestable como medida terapéutica en busca de prevenir estas complicaciones ${ }^{4,7}$.

El tiempo recomendado para la estabilización quirúrgica temprana del tórax es de menos de 10 días $^{10}$, con un periodo ideal entre las primeras 24 a 72 horas de producida la fractura ${ }^{9}$, evitando así la formación de callo óseo, procesos inflamatorios y hematomas graves $^{11}$. Este procedimiento se indica en pacientes hemodinámicamente estables, con falla ventilatoria causada por el tórax inestable, dolor agudo que no cede con el manejo médico y deformidad importante en la pared del tórax que produce alteración de la mecánica ventilatoria ${ }^{1,5,9,11}$. De otro lado, se ha proscrito en pacientes con inestabilidad hemodinámica, traumatismos cerebrales graves, presencia de contusión pulmonar grave o fracturas inestables de la pelvis y la columna vertebral asociadas. En el escenario no agudo, se considera en el $5-10 \%$ de las fracturas costales en las que no ha habido unión después del manejo conservador ${ }^{9}$.

Entre los beneficios de la fijación temprana de las fracturas costales en el tórax inestable se ha descrito la restauración rápida de la mecánica ventilatoria, el reintegro laboral más rápido, una mejor función respiratoria a largo plazo y una disminución de los costos, del dolor crónico, del uso de narcóticos, de la estancia en la $\mathrm{UCl}$ y en el hospital, del riesgo de neumonía asociada al ventilador, del riesgo de deformidad de la pared torácica a largo plazo y de la mortalidad $^{3,5,9}$.

Esto se hace evidente en estudios como el publicado en 2016 por Schuurmans, et al..$^{12}$, en el cual se logró evidenciar la superioridad de la fijación temprana en cuanto a incidencia de neumonía, menor tiempo de soporte ventilatorio, estancia en la $\mathrm{UCl}$, estancia hospitalaria, requerimiento de traqueostomía y reducción de costos, sin encontrar una diferencia 
Tabla 1. Recomendaciones para la fijación temprana del tórax inestable

- El diagnóstico de tórax inestable es clínico y radiológico`.

- La tomografía computarizada de tórax con reconstrucción 3D permite identificar adecuadamente los arcos costales comprometidos y planear el procedimiento quirúrgico $0^{7,13}$.

- Los candidatos a manejo quirúrgico deben ser escogidos cuidadosamente teniendo en cuenta su condición clínica y su respuesta al manejo médico $0^{1,5,9,11}$

- Las indicaciones para la fijación temprana del tórax inestable son:

- Falla ventilatoria causada por el tórax inestable 1,9,11.

- Dolor agudo que no ceda al manejo médico $0^{5,9,11}$.

- Deformidad importante en la pared del tórax que produzca alteración de la mecánica ventilatoria ${ }^{5,9,11}$.

- Las contraindicaciones para la fijación temprana del tórax inestable son:

- Inestabilidad hemodinámica ${ }^{9}$

- Traumatismo cerebral grave ${ }^{9}$.

- Contusión pulmonar ${ }^{9}$.

- Fracturas inestables de la pelvis y la columna vertebral9.

- Los beneficios de la fijación temprana de las fracturas costales en el tórax inestable son:

- Restauración rápida de la mecánica ventilatoria2,5,9.

- Reintegro laboral más temprano $2,9$.

- A largo plazo mejor función respiratoria².

- Disminución en los siguientes ítems: costos, dolor crónico, uso de narcóticos, estancia hospitalaria y en unidad de cuidados intensivos, riesgo de neumonía asociada al ventilador, riesgo de deformidad de la pared torácica a largo plazo y mortalidad 2,5,9.

- Se deben fijar todos los trazos de fractura y las costillas de la $3 .^{a}$ a la $10{ }^{a}$ para brindar mayor estabilidad y mejor resultado en el control del dolor ${ }^{13}$.

importante en cuanto a la mortalidad. Estos hallazgos también se correlacionan con lo publicado por Domínguez, et al. ${ }^{4}$, quienes reportan su experiencia en el manejo de 10 pacientes con tórax inestable que fueron llevados a fijación temprana con un tiempo quirúrgico promedio de 132 minutos, logrando extubarlos a las 48 horas de posoperatorio y con una estancia hospitalaria promedio de 10 días $^{4}$.

La posición del paciente y el abordaje quirúrgico dependen del número de fracturas y de su localización. Usualmente el paciente se ubica en decúbito lateral y la incisión que se realiza es una toracotomía longitudinal o posterolateral, aunque también puede optarse por un abordaje anterolateral, axilar o inframamario con el objetivo de exponer lo mejor posible las fracturas costales ${ }^{1,5}$. Son numerosos los materiales existentes para realizar la fijación, entre los cuales se incluyen placas bloqueadas, materiales biodegradables, placas de Judet, placas en $U$, dispositivos intramedulares y placas anatómicas diseñadas específicamente para fracturas costales ${ }^{1,5,7}$. Siempre se recomienda fijar los arcos costales del tercero al décimo, dado que el undécimo y el duodécimo no aportan a la mecánica ventilatoria, y el primero y el segundo son de difícil acceso ${ }^{13}$.

En el pasado se recomendaba fijar únicamente una fractura costal para dar estabilidad; sin embargo, la evidencia indica que se deben fijar todas las fracturas costales posibles y ambos trazos de la fractura para evitar complicaciones durante el proceso de osificación, como dolor persistente, deformidades y osificaciones incompletas ${ }^{13}$. Actualmente se están implementando nuevas técnicas quirúrgicas asistidas por toracoscopia para la fijación de las fracturas costales, cuyo beneficio principal es poder fijar fracturas de difícil acceso con incisiones menores, como las de la región subescapular y las posteriores. No obstante, esta técnica no cuenta aún con estudios comparativos que demuestren su superioridad ${ }^{14}$.

Por todo lo anterior, basados en la literatura y nuestra experiencia, nos permitimos hacer recomendaciones sobre el diagnóstico, los beneficios, las indicaciones, las contraindicaciones y el manejo intraoperatorio de la fijación temprana del tórax inestable (Tabla 1).

\section{Conclusión}

La reducción abierta y la fijación temprana de las fracturas costales en el contexto del tórax inestable ha demostrado ser un método útil, efectivo y seguro. Logra una reducción de los tiempos de soporte ventilatorio, de la estancia en la UCI, de la incidencia de neumonía y de la incapacidad laboral, entre otras. Se debe realizar siempre y cuando el paciente esté hemodinámicamente estable, no haya respondido al manejo médico adecuado, tenga una mala mecánica ventilatoria que requiera ventilación mecánica y no 
presente lesiones cerebrales graves, contusión pulmonar grave ni inestabilidad de fracturas en la pelvis o en la columna.

\section{Agradecimientos}

Agradecemos especialmente al Dr. Rodolfo Barrios del Río por su compromiso y dedicación.

\section{Conflicto de interés}

Los autores declaran no tener ningún conflicto de intereses.

\section{Responsabilidades éticas}

Protección de personas y animales. Los autores declaran que para esta investigación no se han realizado experimentos en seres humanos ni en animales.

Confidencialidad de los datos. Los autores declaran que han seguido los protocolos de su centro de trabajo sobre la publicación de datos de pacientes.

Derecho a la privacidad y consentimiento informado. Los autores han obtenido el consentimiento informado de los pacientes y/o sujetos referidos en el artículo. Este documento obra en poder de los autores.

\section{Bibliografía}

1. Geoff Vana P, Neubauer DC, Luchette FA. Contemporary management of flail chest. Am Surg. 2014;80:527-35.

2. Dehghan N, de Mestral C, McKee MD, Schemitsch EH, Nathens A. Flail chest injuries: a review of outcomes and treatment practices from the National Trauma Data Bank. J Trauma Acute Care Surg. 2014:76:462-8.

3. Lafferty PM, Anavian J, Will RE, Cole PA. Operative treatment of chest wall injuries: indications, technique, and outcomes. J Bone Joint Surg Am. 2011;93:97-110.

4. Domínguez AF, Velázquez M, Ordóñez CA. Manejo quirúrgico del tórax inestable: experiencia en la Fundación Valle de Lili. Panam J Trauma Critical Care Emerg Surg. 2012;1:215-8.

5. Fowler TT, Taylor BC, Bellino MJ, Althausen PL. Surgical treatment of flail chest and rib fractures. J Am Acad Orthop Surg. 2014;22:751-60.

6. Advanced trauma life support, ATLS ${ }^{\circledR}$, curso avanzado para cirujanos. 10. ${ }^{\text {a }}$ ed. Chicago: American College of Surgeons; 2018.

7. Senekjian L, Nirula R. Rib Fracture fixation indications and outcomes. Crit Care Clin. 2017;33:153-65.

8. Kocher GJ, Sharafi S, Azenha LF, Schmid RA. Chest wall stabilization in ventilator-dependent traumatic flail chest patients: who benefits? Eur J Cardiothorac Surg. 2017;51:696-701.

9. Ribas Milanez de Campos J, White TW. Chest wall stabilization in trauma patients: why, when, and how? J Thorac Dis. 2018;10:S951-62.

10. Freixinet Gilart J, Hernández Rodríguez $H$, Martínez Vallina $P$, Moreno Balsalobre R, Rodríguez Suárez P. Normativa sobre diagnóstico y tratamiento de los traumatismos torácicos. Arch Bronconeumol. 2011:47:41-9.

11. Mitchell JD. Blunt chest trauma: is there a place for rib stabilization? J Thorac Dis. 2017;9(Suppl 3):S211-7.

12. Schuurmans J, Goslings JC, Schepers T. Operative management versus non-operative management of rib fractures in flail chest injuries: a systematic review. Eur J Trauma Emerg Surg. 2017;43:163-8.

13. Marasco S, Saxena P. Surgical rib fixation - technical aspects. Injury. 2015:46:929-32.

14. Pieracci FM. Completely thoracoscopic surgical stabilization of rib fractures: can it be done and is it worth it? J Thorac Dis. 2019;11(Suppl 8):S1061-9. 